\title{
Inactivité physique et nombre d'heures passées devant la télévision chez les adultes autochtones asthmatiques : analyse transversale de l'Enquête auprès des peuples autochtones
}

\author{
N. Doggett, B. Sc. Kin. (1); S. Dogra, Ph. D. (2)
}

Cet article a fait l'objet d'une évaluation par les pairs.

Diffuser cet article sur Twitter

\section{Résumé}

Contexte: Notre analyse visait à déterminer l'association entre l'asthme et le niveau d'activité physique ainsi qu'avec le nombre d'heures d'activités sédentaires chez les adultes autochtones et visait à comprendre l'influence de l'inactivité physique et du nombre d'heures d'activités sédentaires sur l'utilisation des soins de santé par les adultes autochtones asthmatiques.

Méthodologie: Notre analyse a porté sur 20953 répondants adultes de l'Enquête auprès des peuples autochtones de 2006. Nous avons considéré que les répondants étaient atteints d'asthme « actuel » s'ils avaient déclaré avoir reçu un diagnostic d'asthme de la part d'un médecin et s'ils avaient une ordonnance valide pour des médicaments contre l'asthme. Notre définition d'une activité physique insuffisante correspondait au fait de pratiquer moins de 3 heures d'activité physique modérée à intense par semaine, et notre définition d'un nombre élevé d'heures passées devant la télévision correspondait au fait de regarder la télévision pendant plus de 10 heures par semaine. Nous avons évalué l'utilisation des soins de santé à l'aide du nombre de consultations de professionnels de la santé et du nombre d'hospitalisations d'au moins une nuit.

Résultats: Les adultes autochtones asthmatiques étaient plus susceptibles de déclarer un nombre élevé d'heures passées devant la télévision ( $\mathrm{RC}=1,16$; IC : 1,11 à 1,22) et une activité physique insuffisante ( $\mathrm{RC}=1,15$; IC : 1,10 à 1,20) que les non-asthmatiques. Les asthmatiques ayant déclaré un nombre élevé d’heures passées devant la télévision ont signalé plus de consultations de professionnels de la santé au cours des 12 derniers mois ( $\mathrm{RC}=2,59$; IC : 2,34 à 2,87), plus d'hospitalisations d'au moins une nuit au cours de la dernière année $(\mathrm{RC}=1,95$; IC : 1,82 à 2,08) et plus d'hospitalisations d'au moins une nuit au cours des 5 dernières années $(\mathrm{RC}=1,13$; IC : 1,07 à 1,18). Les résultats étaient moins cohérents pour l'activité physique et l'utilisation des soins de santé.

Conclusion: Ces résultats laissent penser que les adultes autochtones asthmatiques sont moins actifs que leurs pairs non asthmatiques et que ce mode de vie est lié à une utilisation accrue des soins de santé. Ces résultats sont importants pour les stratégies de promotion de l'activité physique et de lutte contre le comportement sédentaire ciblant les adultes autochtones asthmatiques.

Mots-clés : activité physique, mode de vie sédentaire, maladie chronique, contrôle de l'asthme

\section{Introduction}

Chez les adultes autochtones au Canada, le fardeau des maladies chroniques est dispro- portionnellement élevé, la mortalité plus importante et l'espérance de vie réduite ${ }^{1}$. Selon plusieurs études, la prévalence de l'asthme est également plus élevée chez

\section{Principales constatations}

- Les adultes autochtones asthmatiques sont plus sédentaires et moins actifs physiquement que leurs pairs non asthmatiques.

- En outre, les adultes autochtones asthmatiques qui déclarent un nombre élevé d'heures passées devant la télévision sont plus susceptibles d'utiliser les services de soins de santé que leurs pairs moins sédentaires.

les adultes métis, inuits et membres des Premières Nations vivant hors réserve (13\% à $14 \%$ ) que chez les adultes non autochtones $(8,6 \%)^{2}$. Un statut socioéconomique faible, un accès limité aux services de soins de santé et une mauvaise observance du traitement expliquent en partie cet écart ${ }^{3}$.

Entre 1998 et 2001, les symptômes liés à l'asthme ont entraîné plus de 80000 hospitalisations ${ }^{4}$. En 2011, le fardeau économique global de l'asthme au Canada, incluant les coûts directs et indirects, se chiffrait à plus de 2 milliards de dollars ${ }^{5}$. Les données sur les hospitalisations associées à l'asthme chez les Autochtones ne sont pas accessibles. Toutefois, une étude longitudinale en Saskatchewan a révélé que les Indiens inscrits âgés de 35 à 64 ans présentaient un risque significativement accru d'hospitalisation liée à l'asthme ${ }^{6}$. De plus, d'après une étude de cohorte rétrospective en Alberta, les Indiens inscrits étaient deux fois plus susceptibles de se rendre au service d'urgence d'un hôpital en raison de symptômes d'asthme ou de

Rattachement des auteurs :

1. School of Recreational Management and Kinesiology, Acadia University, Wolfville (Nouvelle-Écosse), Canada

2. Faculté des sciences de la santé, Kinésiologie, Institut universitaire de technologie de l'Ontario, Oshawa (Ontario), Canada

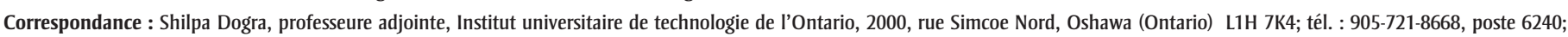
courriel : shilpa.dogra@uoit.ca 
MPOC que les non-Autochtones, mais ils étaient moins susceptibles de consulter un spécialiste et de subir une spirométrie ${ }^{3}$.

Des travaux de recherche ont montré que l'activité physique protège les individus contre la dégradation de leur état de santé et contre diverses affections comme le diabète, l'hypertension, les cardiopathies, l'asthme et l'arthrite, et qu'elle peut aider à prévenir l'évolution de ces maladies ${ }^{7,8}$. Les plus récentes données sur le niveau d'activité physique chez les Autochtones au Canada indiquent que seulement $21 \%$ des membres des Premières Nations vivant dans une réserve sont physiquement actifs $^{9}$, comparativement à 53,8 \% des non-Autochtones ${ }^{10}$. Ce niveau d'activité physique plus faible peut en partie expliquer la fréquence accrue et la moins bonne prise en charge des maladies chroniques dans cette population ${ }^{9}$. L'activité physique régulière est associée à un meilleur conrôle de l'asthme ${ }^{11}$ ainsi qu'à une utilisation réduite des soins de santé ${ }^{12}$. La limitation d'activité est un critère important pour évaluer le contrôle de l'asthme ${ }^{13}$.

Malheureusement, les données concernant les effets d'un comportement sédentaire sur le contrôle de l'asthme sont insuffisantes. D’après certaines études récentes, le comportement sédentaire (défini comme le temps passé en position assise dans les transports, au travail ou pendant les loisirs ${ }^{14}$ ) est un facteur de risque indépendant de morbidité chronique et de mortalité toutes causes confondues ${ }^{15}$. Une grande proportion du temps de loisirs sédentaires se passe devant un écran, en particulier devant la télévision ${ }^{16}$. En fait, le temps passé devant la télévision constitue la plus grande période passée quotidiennement devant un écran chez les adultes canadiens, avec 29 \% d'entre eux ayant déclaré y passer 15 heures ou plus par semaine (plus de 2 heures par jour) en $2007^{17}$. Une étude récente indique également que $64 \%$ des Métis ont déclaré regarder la télévision plus de 6 heures par semaine $^{18}$. Toutefois, les répercussions de ces activités sédentaires sur les résultats liés à l'asthme ne sont pas établies.

$\mathrm{Au}$ Canada, la prévalence de l'asthme est plus élevée ${ }^{2}$, le contrôle de l'asthme semble moins efficace ${ }^{19}$ et l'inactivité physique est plus importante ${ }^{9}$ chez les Autochtones.
Étant donné qu'il y a un lien entre l'activité physique et les effets de l'asthme sur la santé, une meilleure compréhension de l'association entre l'asthme, la pratique régulière d'une activité physique et l'importance des activités sédentaires est essentielle pour réduire les inégalités en matière de santé chez les Autochtones. Notre étude visait ainsi à déterminer l'association entre l'asthme et le niveau d'activité physique ainsi que le nombre d'heures d'activités sédentaires chez l'ensemble des adultes autochtones ainsi que chez chacun des groupes et à comprendre l'influence de l'inactivité physique et du nombre d'heures d'activités sédentaires sur l'utilisation des soins de santé par les adultes autochtones asthmatiques.

\section{Méthodologie}

\section{Données et participants}

Nous avons utilisé l'Enquête auprès des peuples autochtones (EAPA) de 2006 dans le cadre de la présente analyse. L'EAPA est une enquête nationale menée par Statistique Canada auprès d'Autochtones canadiens. Elle aborde des questions comme l'éducation, la langue, l'emploi, le revenu, la santé, la mobilité et le logement chez les Autochtones. La population cible est constituée des membres des Premières Nations, des Métis et des Inuits âgés de 6 ans ou plus, vivant hors réserve en zone rurale, urbaine ou nordique. Toutes les données obtenues dans le cadre de l'EAPA ont été autodéclarées lors d'entrevues effectuées par téléphone ou en personne avec les répondants.

L'échantillon de l'EAPA de 2006 comptait 61041 sujets, qui ont répondu dans une proportion de 80,1 \%. Dans le cadre de notre étude, nous avons utilisé le fichier de microdonnées à grande diffusion ( $\mathrm{n}=24$ 368). Étant donné que notre étude s'intéressait aux adultes, nous avons exclu de notre analyse les 3415 sujets de moins de 20 ans ( $n=20$ 953).

Des renseignements détaillés concernant l'échantillonnage, la collecte de données et la pondération des données de l'EAPA sont fournis dans le guide des concepts et méthodes de l'EAPA de $2006^{20}$.

\section{Variables principales}

\section{Asthme}

Les participants de l'EAPA devaient indiquer s'ils avaient déjà reçu un diagnostic d'asthme de la part d'un médecin. Si oui, ils devaient indiquer s'ils suivaient actuellement un traitement ou s'ils prenaient des médicaments pour traiter leur asthme. Pour nous assurer que les sujets étaient atteints d'asthme " actuel » et pour éviter une classification erronée en raison d'un surdiagnostic ${ }^{21}$, nous avons considéré comme asthmatiques les sujets qui avaient répondu oui aux deux questions. Tous les autres répondants ont été considérés comme non asthmatiques. Cette définition de l'asthme a entraîné l'exclusion de 665 répondants.

\section{Activité physique insuffisante (API)}

Cette variable a été construite à partir de la question de l'Enquête : "Au cours d'une semaine normale, combien de temps passezvous à faire de l'activité physique en dehors du travail qui fait augmenter votre fréquence cardiaque et respiratoire? ». Les choix de réponse étaient les suivants : aucun, de 1 à 2 heures, de 3 à 4 heures, de 5 à 6 heures, de 7 à 10 heures, 11 heures ou plus. Les lignes directrices actuelles en matière d'activité physique pour les adultes (18 à 64 ans) recommandant au moins 150 minutes d'activité aérobique modérée à intense par semaine $e^{22}$, nous avons recodé les réponses en deux catégories : activité physique suffisante ( 3 heures et plus par semaine) et activité physique insuffisante (moins de 3 heures par semaine). Nous avons choisi ce seuil prudent pour limiter la classification erronée de répondants inactifs ayant déclaré des niveaux d'activité physique plus élevés que la réalité. Il n’y a aucune donnée sur la fiabilité ou la validité de cette question de l'EAPA, mais des travaux de recherche antérieurs présentaient des niveaux acceptables de validité (comparativement aux données mesurées par un accéléromètre) et de fiabilité de la déclaration des activités physiques modérées à intenses ${ }^{23,24}$.

\section{Heures passées devant la télévision (HTV)}

Cette variable a été construite à partir de la question de l'Enquête : "Au cours des trois derniers mois, durant une semaine normale, combien de temps avez-vous 
habituellement passé à regarder la télévision? » Les choix de réponse étaient les suivants : aucun, moins de 1 heure, de 1 à 2 heures, de 3 à 5 heures, de 6 à 10 heures, de 11 à 14 heures, de 15 à 20 heures, 20 heures ou plus. Les réponses ont été recodées en deux catégories : nombre élevé d'heures passées devant la télévision (plus de 10 heures par semaine) et faible nombre d'heures passées devant la télévision (10 heures et moins par semaine). D'après les études épidémiologiques actuelles, les activités sédentaires devraient en effet être limitées à moins de 2 heures par jour ou de 14 heures par semaine, et l'on devrait prendre une pause et se lever après avoir passé 30 minutes en position assise ${ }^{25}$. Les HTV forment une grande partie, mais non la totalité, du temps quotidiennement passé en position assise ${ }^{17}$, ce qui fait que notre catégorisation utilise un seuil vraisemblablement prudent, les adultes ayant également d'autres activités sédentaires, passant du temps par exemple devant l'ordinateur ou à lire.

\section{Utilisation des soins de santé}

Les trois variables suivantes ont servi à évaluer l'utilisation des soins de santé : hospitalisation d'au moins une nuit au cours de la dernière année (oui ou non) ou au cours des 5 dernières années (oui ou non) et consultation d'un professionnel de la santé (médecin de famille, omnipraticien, autre médecin ou spécialiste, infirmière ou guérisseur traditionnel métis, inuit ou des Premières Nations, etc.) au cours des 12 derniers mois (oui ou non).

\section{Covariables}

L’une des covariables utilisées dans l'étude était l'identité autochtone, qui comprenait les catégories de réponse suivantes : "identité unique : Indien d'Amérique du Nord seulement », «identité unique : Métis seulement ", " identité unique : Inuit seulement », « identités multiples », « autres identités autochtones », " aucune identité autochtone » et « non-Inuit habitant dans l'Arctique ».

La sélection des participants de l'EAPA s'est effectuée en fonction des réponses à quatre questions de présélection du recensement de 2006 mené par Statistique Canada. Ces questions étaient destinées à recueillir des renseignements à propos de l'origine ethnique, de l'identité autochtone autodéclarée (Indien d'Amérique du Nord, Métis ou Inuit), de l'appartenance à une bande indienne ou à l'une des Premières Nations ainsi que du statut d'Indien inscrit ou visé par un traité. Les sujets ayant déclaré une origine ancestrale autochtone mais non une identité autochtone ont été considérés comme faisant partie de la population d'ascendance autochtone seulement et ont été inclus dans la catégorie « aucune identité autochtone ».

Les asthmatiques des catégories "identité unique : Indien d'Amérique du Nord seulement » $(\mathrm{n}=715)$, " identité unique : Métis seulement » $(n=598)$ et ceux de l'ensemble de l'échantillon (toutes identités confondues, $\mathrm{n}=1$ 830) ont fait l'objet d'une analyse plus poussée. L'analyse de l'ensemble de l'échantillon a fourni des renseignements utiles au sujet de l'ensemble des Premières Nations (hors réserve), des Métis, des Inuits et des autres identités autochtones au Canada. Nous n'avons pas analysé les autres groupes individuellement en raison de la petite taille de leur échantillon, c'est-à-dire Inuit seulement ( $n=118)$, identités multiples $(n=50)$, autre identité autochtone ( $\mathrm{n}=28$ ), aucune identité autochtone $(\mathrm{n}=314)$ et non-Inuit habitant dans l'Arctique ( $\mathrm{n}=7$ ).

Les autres variables utilisées ont fourni des données sociodémographiques et l'état de santé à partir des choix de réponse offerts aux répondants de l'enquête pour ces variables. De plus amples renseignements sur l'interprétation de ces catégories sont disponibles dans le guide de l'utilisateur ${ }^{22}$.

Des études antérieures ont indiqué que l'âge, le sexe et le milieu de résidence sont des covariables liées à l'asthme chez les quatre groupes d'adultes autochtones ${ }^{2}$ et que le milieu de résidence a été associé à l'accessibilité des services de soins de santé et des ressources en matière d'activité physique ${ }^{26}$. Nous avons donc choisi comme covariables démographiques le sexe (homme ou femme) et la catégorie d'âge (20 à 24 ans, 25 à 34 ans, 35 à 44 ans, 45 à 54 ans ou 55 ans et plus), et comme autre covariable le milieu de résidence (zone métropolitaine centrale, zone urbaine, zone rurale ou zone arctique).

Les covariables socioéconomiques étaient le niveau d'éducation du répondant (études primaires ou moins, études secondaires partielles, études secondaires terminées, études postsecondaires non universitaires partielles, études postsecondaires non universitaires terminées, études universitaires partielles, études universitaires terminées) et le revenu total du ménage (moins de 20000 \$; 20000 à 39999 \$; 40000 à 59999 \$; 60000 à 79999 \$; 80000 à 99999 \$; 100000 \$ et plus). Ces covariables ont été incluses parce que les facteurs socioéconomiques ont été associés au degré de santé et de condition physique chez les adultes autochtones ${ }^{27}$.

Le tabagisme (fumeur quotidien, fumeur occasionnel auparavant fumeur quotidien, fumeur occasionnel stable, ex-fumeur auparavant fumeur quotidien, ex-fumeur auparavant fumeur occasionnel, nonfumeur à vie) et l'indice de masse corporelle (IMC) autodéclaré (insuffisance pondérale [IMC inférieur à 18,5], poids normal [IMC : 18,5 à 24,9], surpoids [IMC : 25,0 à 29,9]; obésité de classe I [IMC :30,0 à 34,9], obésité de classe II [IMC : 35,0 à 39,9] ou obésité de classe III [IMC : 40,0 et plus]) ont été inclus parce qu'on sait qu'il s'agit de facteurs de risque pour la santé ${ }^{28}$.

Les catégories de covariables qui comprenaient moins de $5 \%$ de l'échantillon ont été fusionnées avec une catégorie adjacente, sauf dans le cas de l'insuffisance pondérale, qui n’a pas été fusionnée car il s'agit d'une catégorie d'IMC importante et spécifique.

\section{Analyse statistique}

Nous avons eu recours aux fréquences pour décrire les covariables et les résultats et nous les avons converties en pourcentages de l'échantillon. Les chi carrés de Pearson ont servi à déterminer l'existence ou non de différences entre asthmatiques et non-asthmatiques. Dans les cas où il $\mathrm{y}$ avait plus de deux catégories, nous avons utilisé des résidus standardisés ajustés pour déterminer l'emplacement de la différence. Un seuil de $-2,0$ à 2,0 a été utilisé pour détecter la signification. Nous 
nous sommes servis, en vue de comparer les asthmatiques et les non-asthmatiques, d'analyses de régression logistique ajustée à variable simple et à covariables pour évaluer les associations entre l'asthme et l'API ou les HTV dans chaque groupe d'Autochtones et dans l'ensemble de l'échantillon. Les régressions logistiques ont été ajustées pour les covariables suivantes : âge, sexe, identité autochtone, milieu de résidence, revenu, éducation, statut tabagique et IMC. Nous avons eu recours à des modèles de régression logistique simple et ajustée pour évaluer l'association entre les résultats liés à l'utilisation des soins de santé et l'API ou les HTV dans l'échantillon d'adultes asthmatiques.

Toutes les analyses statistiques ont été effectuées à l'aide de la version 21 du logiciel SPSS et la signification statistique a été établie à $\alpha<0,05$. Afin de nous assurer que les estimations étaient adéquates, nous avons appliqué à l'ensemble des données la pondération normalisée de la population fournie par Statistique Canada.

\section{Résultats}

La prévalence de l'asthme dans notre échantillon était de 8,7 \%. La distribution des identités au sein du groupe asthmatique était la suivante : 39,1 \% d'Indiens d'Amérique du Nord, 32,7 \% de Métis, 11,1 \% d'Inuits, de non-Inuits habitant dans l'Arctique ou de personnes ayant déclaré d'autres identités autochtones ou des identités autochtones multiples et 17,2\% de personne ayant déclaré n'avoir aucune identité autochtone. Le tableau 1 présente les caractéristiques de l'échantillon. Des différences significatives ont été observées entre les sujets traités contre l'asthme et les non-asthmatiques pour la plupart des covariables examinées. La proportion de sujets qui n'étaient pas suffisamment actifs physiquement était plus élevée chez les asthmatiques $(56,9 \%)$ que chez les nonasthmatiques (50,9\%). La proportion de sujets qui déclaraient un nombre élevé d'HTV était plus importante chez les asthmatiques $(50,4 \%)$ que chez les nonasthmatiques $(42,9 \%)$.

Dans les modèles non ajustés (tableau 2), les adultes asthmatiques étaient significativement plus susceptibles de déclarer des
HTV élevées et une API, comparativement aux non-asthmatiques. Ceci se vérifiait pour les Indiens d'Amérique du Nord, les Métis et l'ensemble de l'échantillon. Ces associations restaient significatives après ajustement pour l'âge, le sexe, l'identité autochtone, le milieu de résidence, le revenu, l'éducation, le statut tabagique et l'IMC.

La probabilité d'utiliser des soins de santé était plus importante chez les adultes asthmatiques dont le nombre d'HTV était élevé, tant dans le modèle ajusté que le non ajusté (tableau 3). Pour l'API, toutes les associations non ajustées avec l'utilisation des soins de santé étaient significatives. En ce qui concerne les consultations de professionnels de la santé, l'association était négative dans la régression non ajustée, c'est-à-dire que l'API était associée à moins de consultations $(\mathrm{RC}=0,77$; IC : 0,71 à 0,84 ), alors que l'association entre l'API et les hospitalisations d'au moins une nuit au cours de l'année précédente $(\mathrm{RC}=1,20$; IC : 1,13 à 1,27$)$ et des 5 dernières années ( $\mathrm{RC}=1,49$; IC : $1,43$ à 1,56$)$ étaient toutes deux positives. Dans les modèles ajustés pour l'ensemble des covariables, les hospitalisations d'au moins une nuit au cours de la dernière année et des 5 dernières années étaient toutes deux significativement associées avec l'API.

\section{Analyse}

Nous avons analysé les relations entre l'inactivité physique, les HTV l'asthme et l'utilisation des soins de santé à partir d'un échantillon d'adultes de l'EAPA de 2006. Notre premier résultat est que les adultes asthmatiques étaient significativement plus susceptibles de passer davantage d'heures devant la télévision (plus de 10 heures par semaine) et de ne pas faire assez d'activité physique (moins de 3 heures par semaine) que les non-asthmatiques, et ce, pour les Indiens d'Amérique du Nord, pour les Métis ainsi que pour l'ensemble de l'échantillon. Notre deuxième résultat est que les adultes asthmatiques qui ont déclaré passer davantage d'heures devant la télévision utilisaient également davantage les services de soins de santé que ceux qui passaient moins d'heures devant la télévision. De plus, les adultes asthmatiques qui n'étaient pas suffisamment actifs physiquement déclaraient plus d'hospitalisations que ceux qui l'étaient. Même si des études antérieures ont conclu que les asthmatiques sont plus susceptibles de signaler de faibles niveaux d'activité physique ${ }^{29}$ et une utilisation accrue des soins de santé ${ }^{12}$, notre étude est la première à se pencher sur ces relations dans la population autochtone au Canada ainsi que parmi les répondants déclarant différentes identités autochtones, pour qui le risque peut varier en raison d'une multitude d'inégalités en santé ${ }^{30}$.

Notre résultat indiquant que les adultes autochtones asthmatiques sont plus susceptibles de signaler des niveaux inférieurs d'activité physique est cohérente avec les conclusions d'autres études menées auprès d'adultes asthmatiques non autochtones ${ }^{28}$. De nombreux asthmatiques évitent l'activité physique parce qu'ils ont peur d'exacerber leurs symptômes ou de déclencher une bronchoconstriction à l'effort ${ }^{31}$. Toutefois, de nombreuses études ont montré que l'activité physique peut améliorer le contrôle de l'asthme et la qualité de vie liée à l'asthme ${ }^{11,31}$. Dogra et collab. ${ }^{12}$ ont étudié la relation entre l'activité physique et la santé chez 4272 hommes et 6971 femmes atteints d'asthme ayant participé à l'Enquête sur la santé dans les collectivités canadiennes (cycle 2.1). Les données indiquent que l'activité physique était systématiquement associée à une meilleure santé chez les asthmatiques $^{12}$. De plus, il a été démontré que la pratique d'une activité physique régulière structurée améliore le contrôle de l'asthme chez les adultes asthmatiques ${ }^{11}$. Il pourrait donc être avantageux d'ajouter une composante éducative sur l'asthme dans les stratégies de promotion de l'activité physique chez les Autochtones et l'éducation au sujet de l'asthme devrait quant à elle inclure des renseignements sur l'activité physique.

Les résultats de notre étude indiquent également que les Indiens d'Amérique du Nord qui vivent hors réserve, les Métis et l'ensemble de la population autochtone souffrant d'asthme sont plus susceptibles de signaler des HTV élevées que les nonasthmatiques. Il n'existe qu'un petit nombre d'études sur l'association entre l'asthme et les HTV, et elles ont surtout été menées auprès d'enfants. L'une de ces études a par exemple été menée auprès d'enfants qui ne 
TABLEAU 1

Caractéristiques de l'échantillon en fonction de l'asthme autodéclaré, Enquête auprès des peuples autochtones de 2006

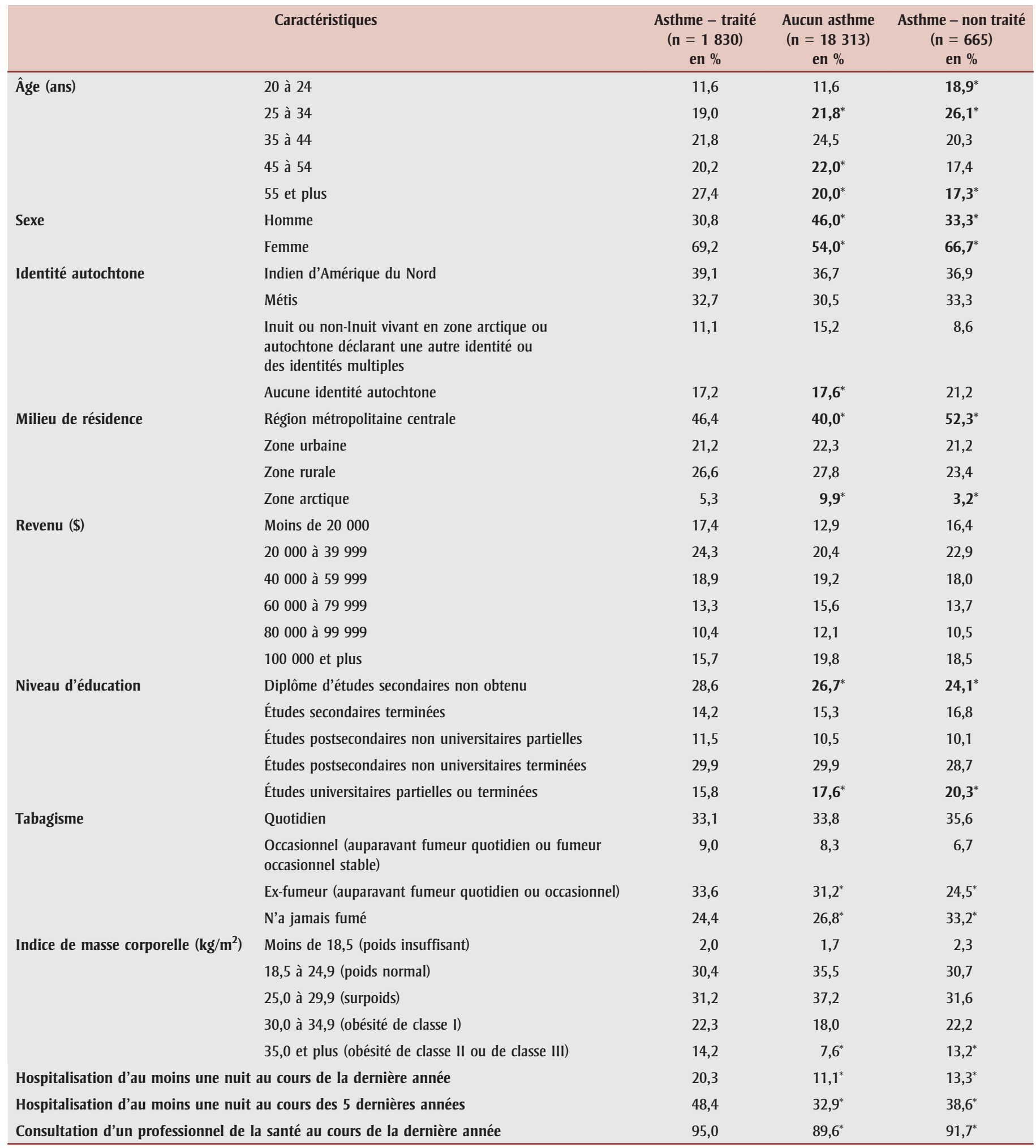

Suite page suivante 
TABLEAU 1 (suite)

Caractéristiques de l'échantillon en fonction de l'asthme autodéclaré, Enquête auprès des peuples autochtones de 2006

\begin{tabular}{|c|c|c|c|c|}
\hline & Caractéristiques & $\begin{array}{c}\text { Asthme - traité } \\
(\mathrm{n}=1830) \\
\text { en } \%\end{array}$ & $\begin{array}{c}\text { Aucun asthme } \\
(n=18313) \\
\text { en } \%\end{array}$ & $\begin{array}{c}\text { Asthme - non traité } \\
\begin{array}{c}(\mathrm{n}=665) \\
\text { en } \%\end{array}\end{array}$ \\
\hline \multirow[t]{4}{*}{ Niveau d'activité physique (heures) } & 0 & 17,8 & 13,9 & 13,4 \\
\hline & 3 à 4 & 20,1 & 21,5 & 23,0 \\
\hline & 5 à 6 & 9,3 & 11,9 & 9,6 \\
\hline & 11 et plus & 5,1 & 6,0 & 8,2 \\
\hline Activité physique & Insuffisante (moins de $3 \mathrm{~h} /$ semaine) & 56,9 & $50,9^{*}$ & $54,8^{*}$ \\
\hline Heures devant la télévision & Sédentaire (plus de $10 \mathrm{~h} /$ semaine) & 50,4 & $42,9^{*}$ & $42,6^{*}$ \\
\hline
\end{tabular}

${ }^{*} p<0,05$.

présentaient pas de respiration sifflante à l'âge de 3,5 ans, dans le cadre d'une étude longitudinale prospective de 7,5 ans qui intégrait des données sur les heures passées devant la télévision ${ }^{32}$. Les résultats ont révélé que les enfants qui avaient regardé la télévision en moyenne 2 heures ou plus par jour pendant la période de 7,5 ans étaient deux fois plus susceptibles de devenir asthmatiques avant l'âge de 11,5 ans $^{32}$. De plus, un grand nombre d'effets néfastes, notamment l'obésité, les troubles de comportement et une baisse du rendement scolaire, sont associés à un temps excessif passé devant un écran ${ }^{33}$, et leur prévalence est plus forte chez les enfants asthmatiques ${ }^{34}$. Des données probantes récentes ont également indiqué qu'un comportement sédentaire, dont des HTV élevées, est un facteur de risque important de cardiopathie et de mortalité toutes causes confondues, indépendamment de l'inactivité physique ${ }^{35}$. Étant donné que les Autochtones sont déjà plus à risque en ce qui concerne bon nombre de ces effets néfastes ${ }^{1}$, le nombre élevé d'HTV déclaré par les adultes autochtones asthmatiques dans cette étude révèle une cible potentielle pour les futures stratégies de promotion de la santé.

Des études ont montré qu'au Canada, les Autochtones se rendent à l'hôpital plus de deux fois plus fréquemment que leurs pairs non autochtones ${ }^{3}$. Notre étude a révélé que chez les adultes asthmatiques qui ont déclaré un nombre élevé d'HTV, l'utilisation des soins de santé était plus importante. Cela n'est pas surprenant, étant

TABLEAU 2

Heures devant la télévision et inactivité physique chez les Indiens d'Amérique du Nord et les Métis ainsi que dans l'ensemble de l'échantillon d'Autochtones (20 ans et plus), Enquête auprès des peuples autochtones de 2006

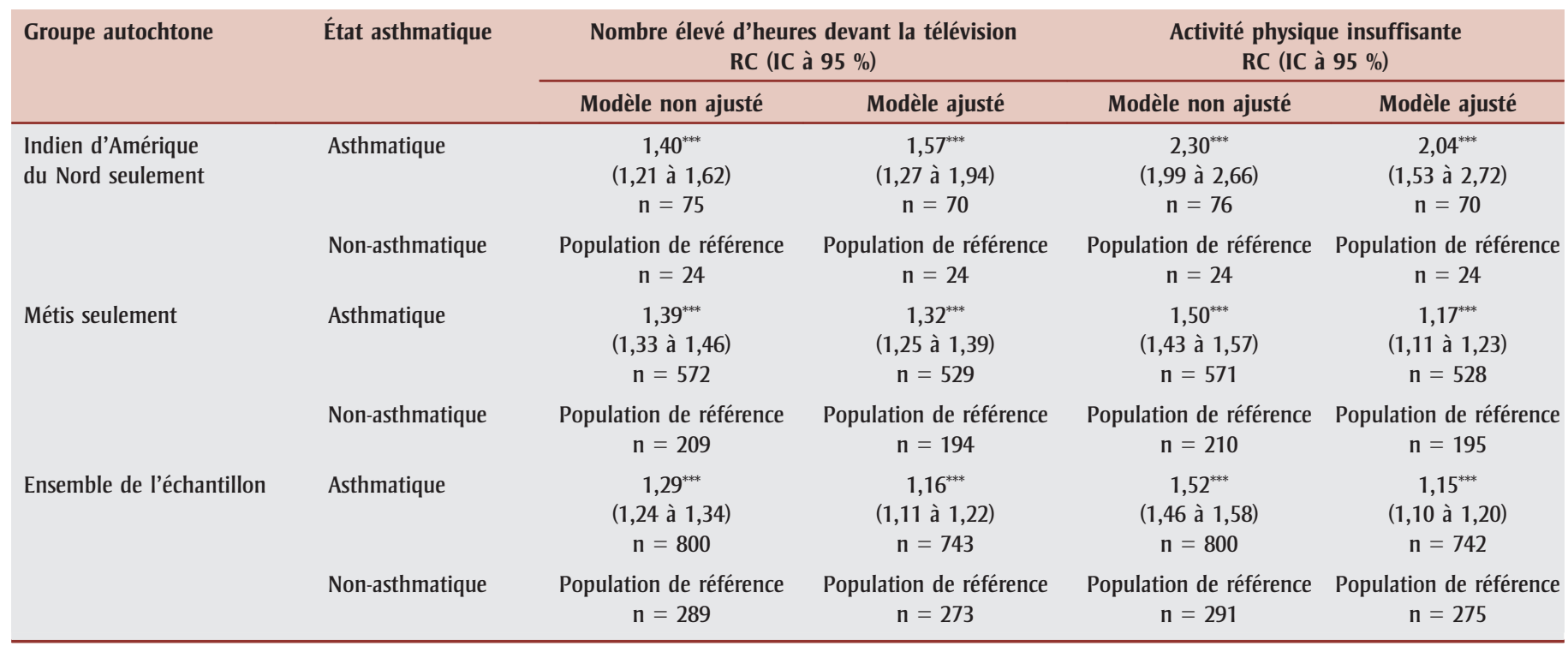

Abréviations : IC, intervalle de confiance; IMC, indice de masse corporelle; RC, rapport de cotes.

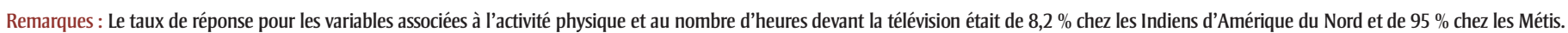
Ajustement pour l'âge, le sexe, le milieu de résidence, le revenu, le niveau d'éducation, le tabagisme et l'IMC.

*** $p<0,001$. 
TABLEAU 3

Association entre l'utilisation des soins de santé et l'inactivité physique ou le nombre d'heures devant la télévision chez les adultes autochtones (20 ans et plus) asthmatiques, Enquête auprès des peuples autochtones de 2006

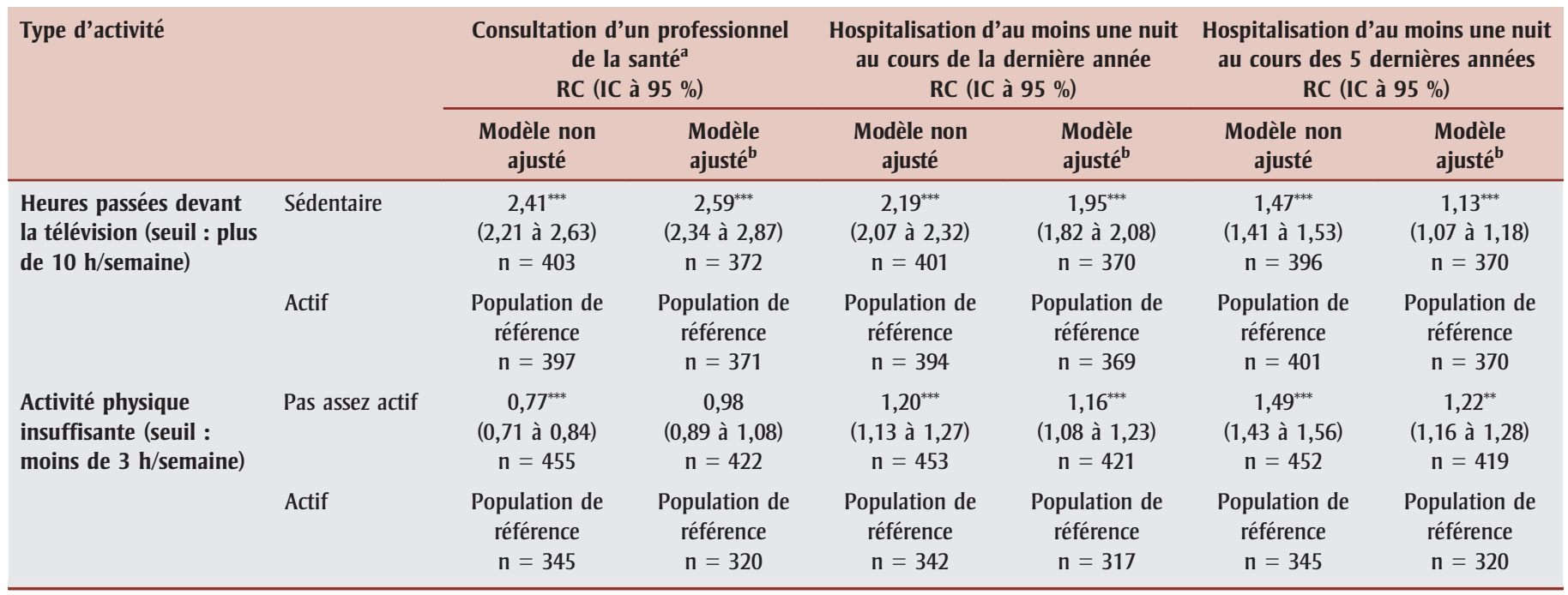

Abréviations : IC, intervalle de confiance; RC, rapport de cotes.

a Médecins de famille, omnipraticiens, autres médecins ou spécialistes, infirmières ou guérisseurs traditionnels métis, inuits ou des Premières Nations, etc.

b Ajustement pour l'âge, le sexe, l'identité autochtone, l'emplacement géographique, le revenu, le niveau d'éducation, le tabagisme et l'indice de masse corporelle.

** $p<0,01$.

*** $p<0,001$.

donné la multitude d'effets néfastes pour la santé associés à des HTV élevées ${ }^{31-33}$. De même, chez les adultes asthmatiques qui n'étaient pas suffisamment actifs, le risque d'hospitalisation d'au moins une nuit au cours de la dernière année était significativement accru. Ce résultat est cohérent avec les résultats antérieurs de l'Enquête sur la santé dans les collectivités canadiennes, qui indiquaient que les adultes asthmatiques physiquement actifs utilisaient moins les services de santé que les adultes asthmatiques inactifs, c'est-à-dire que leur asthme était mieux contrôlé ${ }^{12}$. Il est important de noter que l'association entre inactivité physique et utilisation des soins de santé était moins cohérente et qu'aucune signification statistique n'avait été observée pour les consultations de professionnels de la santé. Les données contradictoires associées à l'inactivité physique et à l'utilisation des soins de santé, en particulier les consultations d'un professionnel, peuvent avoir été faussées par l'asthme à l'effort. Par exemple, des adultes asthmatiques actifs pourraient avoir consulté des professionnels de la santé afin de s'assurer d'avoir les médicaments nécessaires pour prévenir l'inactivité associée aux symptômes de l'asthme. Dans les études ultérieures, il faudrait envisager d'utiliser des mesures plus objectives du contrôle de l'asthme et tenir compte de l'asthme à l'effort.

De plus en plus de données indiquent que les effets sur la santé du temps passé devant un écran sont indépendants de l'activité physique ${ }^{35}$. D'après notre étude descriptive préliminaire, il est difficile de déterminer si le temps consacré à une activité sédentaire a plus d'influence que l'inactivité physique sur les effets associés à l'asthme dans cette population. Il ne fait aucun doute que de plus amples travaux sont nécessaires. Quoi qu'il en soit, les données de notre étude indiquent clairement que si les adultes autochtones asthmatiques augmentaient leur activité physique et diminuaient leurs HTV, cela pourrait améliorer le contrôle de leur asthme, réduire les coûts des soins de santé et améliorer leur qualité de vie.

\section{Points forts et limites}

Les points forts de cette analyse sont la grande taille de notre échantillon, qui a permis de procéder à l'analyse pour deux identités autochtones distinctes, et le nombre de variables disponibles dans l'EAPA qui ont pu faire l'objet d'un ajustement dans les modèles de régression. Les résultats de notre étude doivent cependant être interprétés à la lumière des limites ci-dessous.

Premièrement, les données utilisées dans cette étude étaient transversales, ce qui fait qu'une causalité inverse ne peut être exclue. Deuxièmement, certaines covariables non mesurées dans l'étude, par exemple l'asthme à l'effort, peuvent constituer un facteur de confusion. Troisièmement, toutes les données étaient autodéclarées, ce qui peut avoir faussé la classification, particulièrement pour l'activité physique, les comportements sédentaires et l'IMC. En ce qui concerne l'activité physique et le comportement sédentaire, il a été démontré que la mémoire des répondants a une validité et une fiabilité acceptables lorsqu'on utilise un questionnaire de rappel des activités effectuées au cours des 7 derniers jours ${ }^{23,24}$, et que l'IMC autodéclaré peut permettre de prédire avec précision l'IMC mesurés ${ }^{36}$. Toutefois, les données mesurées objectivement permettent de mieux prédire les effets sur la santé ${ }^{37}$ que les données autodéclarées. Des seuils prudents ont donc été choisis dans cette étude afin de réduire ce biais potentiel. Les travaux de recherche ultérieurs devront utiliser des données mesurées objectivement ou des outils valides et fiables pour mesurer ces variables. 
Quatrièmement, l'échantillon ne comprenait pas de membres des Premières Nations vivant sur une réserve, et l'échantillon n'était pas assez important pour effectuer des analyses individuelles pour toutes les identités autochtones, deux éléments qui peuvent limiter la généralisation potentielle des résultats. Il faut noter que l'EAPA ne contient pas les renseignements nécessaires pour analyser en détail l'accessibilité des soins de santé pour chacune de ces identités. D'autres études devraient se pencher sur l'asthme chez les Premières Nations, les Métis et les Inuits séparément pour mieux comprendre les associations au sein de ces populations en particulier. Cinquièmement, comme l'EAPA ne contient pas de données sur le contrôle de l'asthme, des résultats liés à l'utilisation des soins de santé ont servi d'indicateur. D'autres études devraient évaluer directement l'association entre l'API, les HTV et le contrôle de l'asthme chez les adultes autochtones asthmatiques, qu'ils vivent dans une réserve ou hors réserve. Enfin, la présente analyse était la première à examiner de telles associations chez les adultes autochtones. D'autres études devraient être menées pour examiner les modificateurs d'effets potentiels et déterminer si les associations sont influencées par d'autres variables, notamment le tabagisme et la consommation de médicaments.

\section{Conclusion}

D’après les données de l'EAPA, les adultes autochtones asthmatiques sont plus sédentaires et moins actifs physiquement que leurs pairs non asthmatiques. En outre, les adultes autochtones asthmatiques qui déclaraient passer de nombreuses heures devant la télévision étaient plus susceptibles d'utiliser des services de santé que leurs pairs moins sédentaires. Ces résultats ont des répercussions importantes sur l'éducation à faire concernant l'asthme à l'effort chez les populations autochtones au Canada.

\section{Conflit d'intérêts}

Les auteurs ne signalent aucun conflit d'intérêts. Les auteurs sont seuls responsables du contenu et de la rédaction de cet article.

\section{Références}

1. King M. Maladies chroniques et taux de mortalité au sein des populations autochtones du Canada : tirer profit des connaissances. Maladies chroniques au Canada. 2010;31(1):3-4.

2. Change HJ, Beach J, Senthilselvan A. Prevalence and risk factors of asthma in off-reserve Aboriginal children and adults in Canada. Can Respir J. 2012;16(6):e68e74.

3. Crighton E, Wilson K, Senecal S. The relationship between socio-economic and geographic factors and asthma among Canada's Aboriginal populations. Int $\mathrm{J}$ Circumpolar Health. 2010;69(2):138-150.

4. Chen Y, Johansen H, Thillaiampalam S, Sambell C. L'asthme. Rapports sur la santé. 2005;16(2):45-49.

5. Wilson C. Présentation par The Canadian Lung Association/L'Association pulmonaire du Canada lors des consultations prébudgétaires du Comité permanent des finances de la Chambre des communes. Ottawa (Ont.) : l'Association pulmonaire; 2011.

6. Senthilselvan A, Habbick BF. Increased asthma hospitalizations among registered Indian children and adults in Saskatchewan, 1970-1989. J Clin Epidemiol. 1995;48(10): 1277-1283.

7. Warburton DE, Katzmarzyk PT, Rhodes RE, Shephard RJ. Lignes directrices éclairées par des données probantes sur l'activité physique à l'intention des Canadiens adultes. Physiologie appliquée, nutrition et métabolisme. 2007;32(S2F):S17-74.

8. Humphreys BR, McLeod L, Ruseski JE. Physical activity and health outcomes: evidence from Canada. Health Econ. 2014; 23(1):35-54.

9. Young TK, Katzmarzyk PT. L’activité physique chez les Autochtones au Canada. Physiologie appliquée, nutrition et métabolisme. 2007;32 Suppl 2F:S165-178.
10. Statistique Canada. Activité physique durant les loisirs, 2011 [Internet]. Ottawa (Ont.) : Statistique Canada [consultation le 21 novembre 2013]. Consultable en ligne à la page : http://www.statcan.gc.ca/pub /82-625-x/2012001/article/11667-fra.htm.

11. Dogra S, Kuk JL, Baker J, Jamnik V. Exercise is associated with improved asthma control in adults. Eur Respir J. 2011;37:318-323.

12. Dogra S, Baker J, Ardern CI. The role of physical activity and body mass index in the use of adults with asthma. Ann Allergy Asthma Immunol. 2009;102(6):462-468.

13. Cochrane LM, Clark CJ. Benefits and problems of a physical training programme for asthmatic patients. Thorax. 1990;45(5):345351.

14. Owen N, Healy GN, Matthews CE, Dunstan DW. Too much sitting: the populationhealth science of sedentary behaviour. Exerc Sport Sci Rev. 2010;38(3):105-113.

15. Thorp A, Owen N, Neuhaus M, Dunstan D. Sedentary behaviors and subsequent health outcomes in adults a systematic review of longitudinal studies, 1996-2011. Am J Prev Med. 2011;41(2):207-215.

16. Tremblay MS, Colley RC, Saunders TJ, Healy GN, Own N. Physiological and health implications of a sedentary lifestyle. Appl Physiol Nutr Metab. 2010:35(6):725-740.

17. Shields M, Tremblay M. Comportements sédentaires et obésité. Rapports sur la santé. 2008;19(2):21-33.

18. Janz T, Seto J, Turner A. Enquête auprès des peuples autochtones de 2006: un aperçu de la santé de la population métisse. Ottawa (Ont.): Statistique Canada, Division de la statistique sociale et autochtone; 2009. (n॰89-637-X, 004)

19. Sin DD, Wells H, Svenson LW, Man SF. Asthma and COPD among aboriginals in Alberta, Canada. Chest. 2002;121(6):18411846.

20. Statistique Canada. L'Enquête auprès des peuples autochtones de 2006 : guide des concepts et méthodes. Ottawa (Ont.) : Statistique Canada, Division de la statistique sociale et autochtone; 2009. (n॰89637-X, 003) 
21. Aaron SD, Vandemheen KL, Boulet LP et collab. Overdiagnosis of asthma in obese and nonobese adults. CMAJ. 2008;179(11):11211131.

22. Société canadienne de physiologie de l'exercice (SCPE). Directives canadiennes en matière d'activité physique [Internet]. Ottawa (Ont.) : SCPE; 2011. PDF téléchargeable à partir du lien : http://www.csep.ca/ CMFiles/Guidelines/CSEP_PAGuidelines_ adults_fr.pdf

23. Craig CL, Marshall AL, Sjostrom $M$ et collab. International physical activity questionnaire: 12 -country reliability and validity. Med Sci Sports Exerc. 2003;35(8):13811395.

24. Zuazagoitia A, Montoya I, Grandes G et collab. Reliability and validity of the 7-day Physical Activity Recall interview in a Spanish population. Eur J Sport Sci. 2014;14(1):S361-368.

25. Owen N, Sugiyama T, Eakin EE, Gardiner PA, Tremblay MS, Sallis JF. Adults' sedentary behavior determinants and interventions. Am J Prev Med. 2011;41(2):189-196.

26. Sibley LM, Weiner JP. An evaluation of access to health care services along the rural-urban continuum in Canada. BMC Health Serv Res. 2011;11(20):1-11.

27. Garner R, Carrière G, Sanmartin C; équipe de recherche de l'Initiative sur les données longitudinales administratives et sur la santé. La santé des adultes chez les Premières Nations vivant hors réserve, les Inuits, et les Métis au Canada : l'incidence du statut socioéconomique sur les inégalités en matière de santé. Ottawa (Ont.) : Statistique Canada, Division de l'information et de la recherche sur la santé; 2010. (n॰ 82-622-X, 004)

28. Koster A, Leitzmann MF, Schatzkin A et collab. The combined relations of adiposity and smoking on mortality. Am J Clin Nutr. 2008;88(5):1206-1212.

29. Ford ES, Heath GW, Mannino DM, Redd SC. Leisure-time physical activity patterns among US adults with asthma. Chest. 2003;124(2):432-437.
30. Lix LM, Bruce S, Sarkar J, Young TK. Facteurs de risque et problèmes de santé chroniques chez les Autochtones et les nonAutochtones. Rapports sur la santé. 2009; 20(4):23-32.

31. Mancuso C, Sayles W, Robbins L et collab. Barriers and facilitators to healthy physical activity in asthma patients. J Asthma. 2006;43(2):137-143.

32. Sherriff A, Maitra A, Ness AR, Mattocks C, Riddoch C, Reilly JJ. Association of duration of television viewing in early childhood with the subsequent development of asthma. Thorax. 2009;64(4):321-325.

33. American Academy of Pediatrics: Committee on Public Education. American Academy of Pediatrics: children, adolescents, and television. Pediatrics. 2001;107(2):423-426.

34. Conn KM, Hernandez T, Puthoor P, Fagnano M, Halterman JS. Screen time use among urban children with asthma. Acad Pediatr. 2009;9(1):60-63.

35. Stamatakis E, Hamer M., Dunstan D. Screen-based entertainment time, all-cause mortality, and cardiovascular events: population-based study with ongoing mortality and hospital events follow-up. J Am Coll Cardiol. 2011;57(3):292-299.

36. Scribani M, Shelton J, Chapel D et collab. Comparison of bias resulting from two methods of self-reporting height and weight: a validation study. JRSM Open. 2014;5(6): 2042533313514048.

37. Shephard RJ. Objective vs. self-reported physical activity and sedentary time: effects of measurement method on relationships with risk biomarkers. Br J Sports Med. 2003; 37:197-206. 